\title{
ALICE @ LHC: Status and Highlights
}

\author{
Alberica Toia ${ }^{1,2, \star}$ \\ ${ }^{1}$ Goethe University of Frankfurt \\ ${ }^{2} \mathrm{GSI}$ Helmholzzentrum fuer Schwerionenforschung
}

\begin{abstract}
ALICE is the dedicated heavy-ion experiment at the Large Hadron Collider at CERN. After a two-year long shutdown, the LHC restarted its physics programme in June 2015 with proton-proton collisions at $\sqrt{s}=13 \mathrm{TeV}$ and $\mathrm{Pb}-\mathrm{Pb}$ collisions at $\sqrt{s_{\mathrm{NN}}}$ $=5.02 \mathrm{TeV}$, the highest centre-of-mass energy ever reached in laboratory. Recent results and future perspective for ALICE will be presented.
\end{abstract}

\section{Introduction}

The harvest of the results collected in heavy ion collisions during the first Run of LHC operations demonstrate the formation of a deconfined phase of matter, the quark-gluon plasma (QGP), at high temperature and energy density [1]. The higher centre-of-mass energy achieved in Run 2, as well as the increased luminosity, offer the possibility to further constrain the fundamental properties of the QGP. This paper will briefly review some of the most recent results of the ALICE experiment and their impact in the characterization of the QGP.

\section{Measuring the collectivity of the system}

\subsection{The energy density}

The charged particle multiplicity density is a measure of the initial energy density and provides information on the dynamics of the soft particle production and its relation to the initial collision geometry. ALICE measured the pseudorapidity density of charged particles measured at central rapidity $(|\eta|<0.5)$, for $\mathrm{pp}$ and $\mathrm{Pb}-\mathrm{Pb}$ collisions at the LHC top energies, divided by the average number of participating nucleon pairs, $\left\langle N_{\text {part }}\right\rangle / 2$, calculated with the MC-Glauber [2]. It is shown in Fig.1 together with a compilation of results at different collider energies. The new measurement at an increased energy allows to probe the increased importance of hard scatterings proportional to the number of binary collisions. The data confirms the trend established by lower energy data of a much stronger rise than the one measured for $\mathrm{pp}$ and even for $\mathrm{p}-\mathrm{Pb}$ collisions, indicating that the particle production in $\mathrm{AA}$ is not solely related to the multiple collisions undergone by the participants. The multiplicity shows a strong dependence on the centrality (Fig.1 right) and a smooth trend towards the value measured in minimum bias $\mathrm{p}-\mathrm{Pb}$ collisions. The centrality dependence of $\mathrm{d} N_{\mathrm{ch}} / \mathrm{d} \eta$ is nearly identical to that measured at $\sqrt{s_{\mathrm{NN}}}=2.76 \mathrm{TeV}$, which showed to be very similar to those measured at RHIC, pointing to a similar mechanism of particle production independent of energy.

^e-mail: alberica.toia@cern.ch 

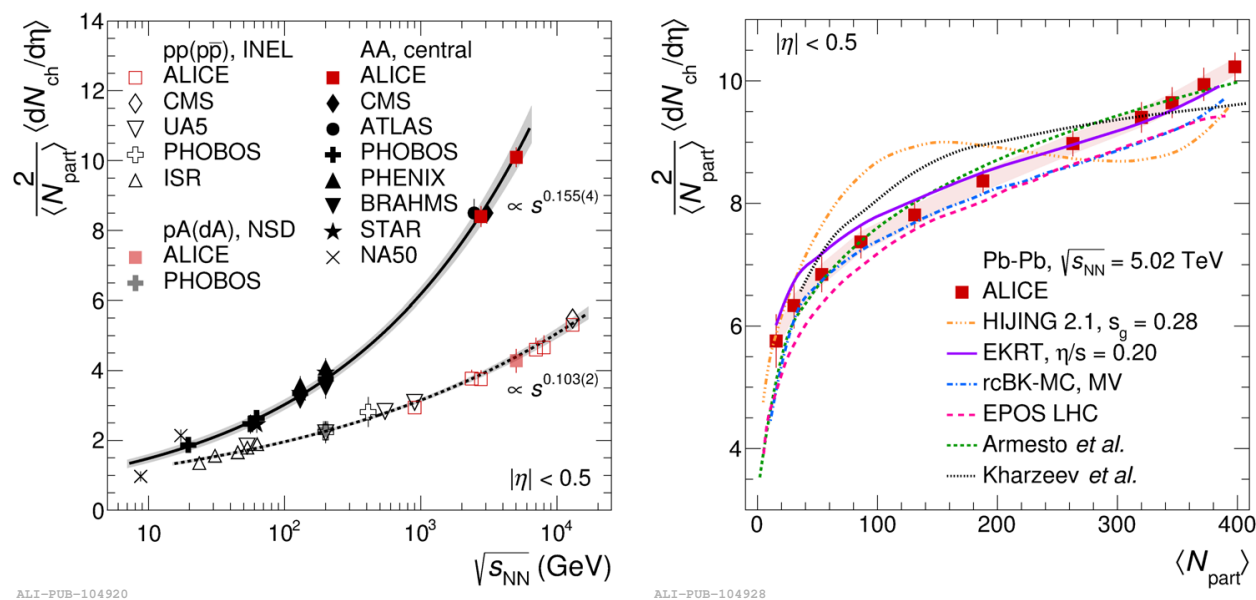

Figure 1. Left: Values of $\frac{2}{\left\langle N_{\mathrm{part}}\right\rangle}\left\langle\mathrm{d} N_{\mathrm{ch}} / \mathrm{d} \eta\right\rangle$ for $\mathrm{pp}, \mathrm{p} \overline{\mathrm{p}}$ and central AA collisions as a function of $\sqrt{s_{\mathrm{NN}}}$. The $s$ dependences of the AA and $\mathrm{pp}(\mathrm{p} \overline{\mathrm{p}})$ collision data are well described by the functions $s_{\mathrm{NN}}^{0.155}$ (solid line) and $s_{\mathrm{NN}}^{0.103}$ (dashed line), respectively. Right: The $\frac{2}{\left\langle N_{\mathrm{part}}\right\rangle}\left\langle\mathrm{d} N_{\mathrm{ch}} / \mathrm{d} \eta\right\rangle$ for $\mathrm{Pb}-\mathrm{Pb}$ collisions at $\sqrt{s_{\mathrm{NN}}}=5.02 \mathrm{TeV}$ in the centrality range $0-80 \%$, as a function of $N_{\text {part }}$ in each centrality class.

\subsection{The viscosity}

An important measurement to test the collectivity of the system is the anisotropic flow, which assess if and to which extend the initial spatial anisotropy of the overlap region of colliding nuclei is translated into an anisotropy in momentum space via interactions of produced particles. It is therefore sensitive to the initial collision geometry and transport mechanism and therefore provides a measurement of the collectivity of the medium.

Figure 2 presents the centrality dependence of $\mathrm{v}_{2}, \mathrm{v}_{3}$ and $\mathrm{v}_{4}$, integrated over $0.2<p_{\mathrm{T}}<5.0 \mathrm{GeV} / \mathrm{c}$, for 2.76 and $5.02 \mathrm{TeV} \mathrm{Pb}-\mathrm{Pb}$ collision, with the corresponding ratios on the lower panels. The data is compared to the predictions from hydrodynamic models, which combine the changes in initial spatial anisotropy and the hydrodynamic response [3]. The predictions are compatible with the measured anisotropic flow coefficients. At the same time, a different hydrodynamic calculation, which employs both constant and temperature dependent viscosity can also describe the increase in anisotropic flow measurements of $\mathrm{v}_{2}$. In particular, among the different scenarios, the increase from the two energies, rather moderate for $\mathrm{v}_{2}$ and $\mathrm{v}_{3}$ and more pronounced for $\mathrm{v}_{4}$, but independent on centrality, seem to favor a constant viscosity going from 2.76 to $5.02 \mathrm{TeV} \mathrm{Pb}-\mathrm{Pb}$ collisions.

The measurements of $p_{\mathrm{T}}$-differential flow are more sensitive to initial conditions and viscosity, and are expected to provide important information to constrain further details of the theoretical calculations, e.g. determination of radial flow and freeze-out conditions. Figure 2 presents the flow coefficient v2 as a function of transverse momentum for the $0-5 \%$ and $30-40 \%$ centrality classes. The results are similar to the corresponding measurements at $2.76 \mathrm{TeV}$, indicating that the increase of the $p_{\mathrm{T}}$-integrated flow can be attributed to an increase of mean transverse momentum. While in the midcentral class $\mathrm{v} 2$ remains higher than $\mathrm{v} 3$, with no crossing of the different order flow coefficients, in the top-most central events $\mathrm{v} 3$ becomes larger than v2 at $p_{\mathrm{T}}>2 \mathrm{GeV} / \mathrm{c}$, while v4 is compatible with v2, within uncertainties. 

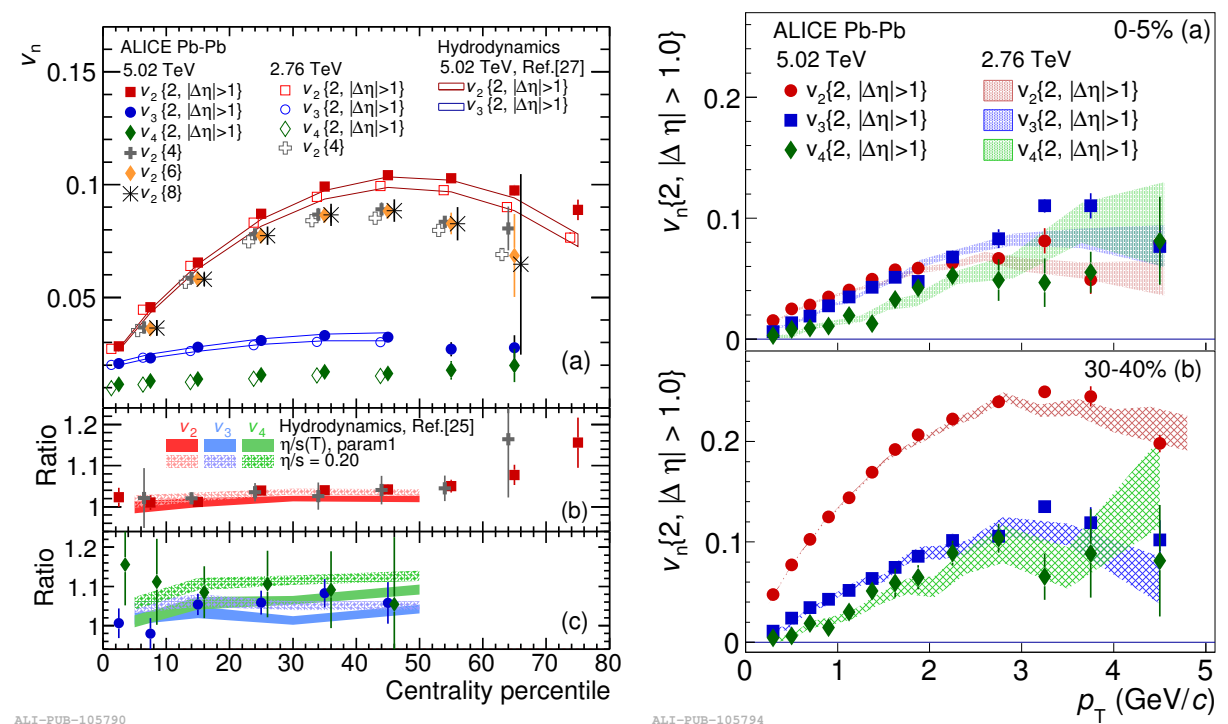

Figure 2. Left: Anisotropic flow $\mathrm{v}_{n}$ integrated over $0.2<p_{\mathrm{T}}<5.0 \mathrm{GeV} / \mathrm{c}$, as a function of event centrality. The ratios between $\mathrm{Pb}-\mathrm{Pb}$ collisions at $5.02 \mathrm{TeV}$ and $2.76 \mathrm{TeV}$, are presented in the lower panels. Various hydrodynamic calculations are also presented. Right: $\mathrm{vn}\left(p_{\mathrm{T}}\right)$ for $0-5 \%$ and $30-40 \%$ centrality classes.

\section{Understanding the energy loss}

Hard processes are those which occur with high momentum transfer, where the production rate in pp collisions can be calculated with pQCD techniques using the universality of PDF and FF and the factorization theorem. In AA collisions the produciton rate is expected to scale with the number of elementary nucleon-nucleon collisions $N_{\text {coll }}$, typically calculated with Glauber models. Any deviation from this scaling is experimentally quantified by the nuclear modification factor, $R_{\mathrm{AA}}$, defined as the ratio of the yield in $\mathrm{Pb}-\mathrm{Pb}$ to that in $\mathrm{pp}$, scaled by $N_{\text {coll }}$. Experimental observables connected to hard processes are hadrons with high $p_{\mathrm{T}}$, jets, or hadrons from open heavy flavour.

\subsection{The energy loss for leading hadrons}

ALICE has measured the transverse momentum spectra of primary charged particles at various collision energies. In Run 2 the top LHC energy of $13 \mathrm{TeV}$ was reached in pp and $5.02 \mathrm{TeV}$ for $\mathrm{Pb}-\mathrm{Pb}$ collsions. Figure 3 presents the measured $p_{\mathrm{T}}$ spectrum and its comparison with calculations with various MC models, i.e. PYTHIA 6 (Perugia-2011), PYTHIA 8 (Monash-2013) and EPOS LHC [4]. These event generators, benefitting from the tuning performed on the LHC data in Run 1, describe the $p_{\mathrm{T}}$ spectrum reasonably well, although not in detail. As expected, and reasonably reproduced by MC models, the spectrum is significantly harder at $\sqrt{s}=13 \mathrm{TeV}$ than at $\sqrt{s}=7 \mathrm{TeV}$. We have also measured the $p_{\mathrm{T}}$ spectra in $\mathrm{Pb}-\mathrm{Pb}$ collisions, for various centrality classes and we have compared it to the spectrum measured at the reference energy of $5.02 \mathrm{TeV}$ in pp scaled by the corresponding number of binary collisions calculated for each centrality class with a Glauber Monte Carlo model. We want to point out the reconstruction and track selection is very much improved with respect to the Run 1 analyses leading to a reduced systematic uncertainties. Moreover a much larger statistics is recorded 

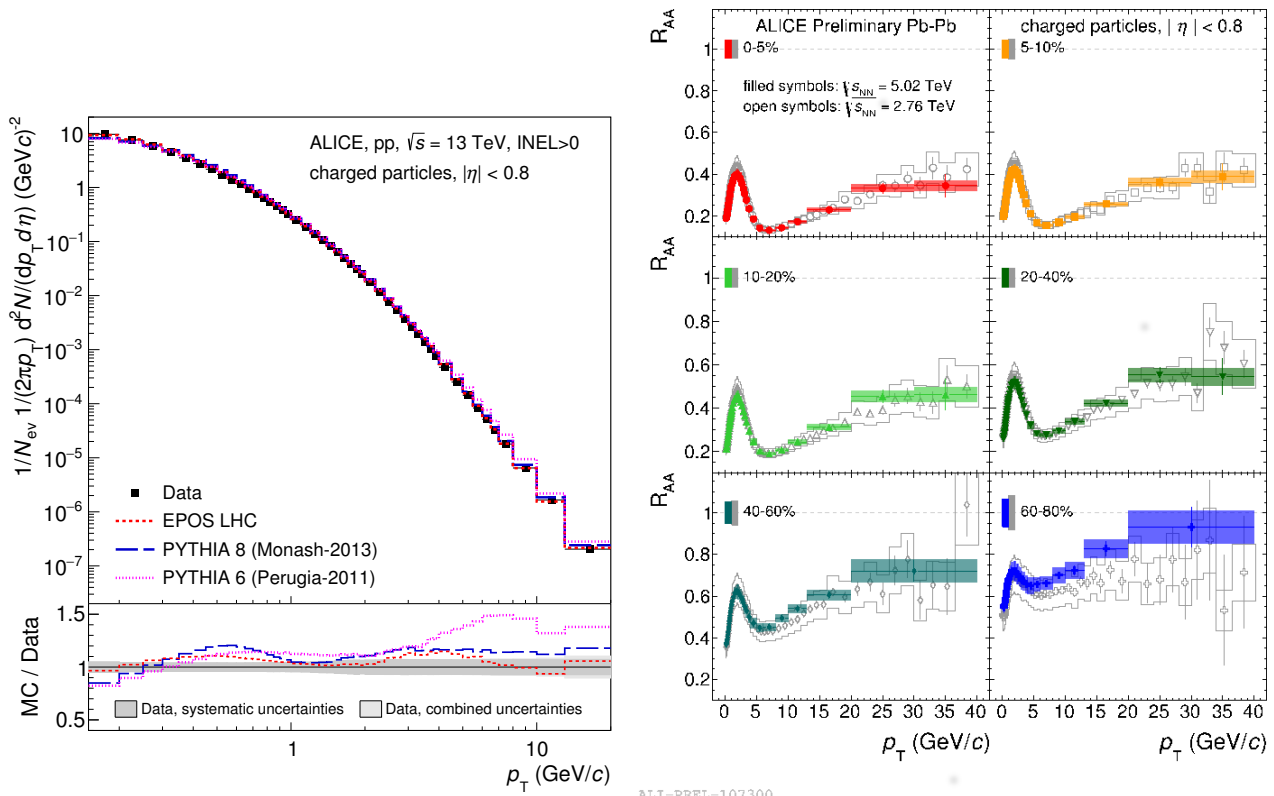

Figure 3. Left:Invariant charged-particle yield as a function of $p_{\mathrm{T}}$ for ppcollisions at $\sqrt{s}=13 \mathrm{TeV}$, compared to Monte Carlo calculations. Right: Nuclear modifications calculated for different classes of centrality. The filled symbols represent the measurement at $\sqrt{s_{\mathrm{NN}}}=5.02 \mathrm{TeV}$ while the open symbols stand for the measurement at $\sqrt{s_{\mathrm{NN}}}=2.76 \mathrm{TeV}$.

for $\mathrm{Pb}-\mathrm{Pb}$ and $\mathrm{pp}$ which is currently under reconstruction. When comparing $\mathrm{Pb}-\mathrm{Pb}$ to pp spectra a clear strong modification of the spectral shape is visible. It has a minimum at $6 \mathrm{GeV}$ followed by a slow increase. The suppression has a strong centrality dependence, and looks very similar to that one measured at $2.76 \mathrm{TeV}$. Considering that the pp spectrum is harder at the higher energy, this similarity of the $R_{\mathrm{AA}}$ may point to a hotter and denser medium produced at the higher centre-of-mass energy. While these results are in agreement with the expectation for models describing energy loss in the medium, improved measurements and detailed comparison with the lower energy will allow to cancel some of the uncertainties and therefore put stronger constrain on the transport properties of the medium.

\subsection{The flavour dependence of energy loss}

Because of their large mass, charm and beauty quark can be produced only in parton scattering with large momentum transfer. while their measurement in pp collisions provides an important test of the factorization hypothesis and the theoretical models based on PQCD, in AA collisions it allows to test the quenching properties of the medium. A milder energy loss is expected for hadrons with charm and beauty than the light hadrons which mostly come from gluon fragmentation. Also the so-called dead cone effect predicts a suppressed gluon radiation at small angles for quarks with large mass at low- $p_{\mathrm{T}}$. The different energy loss, larger for light hadrons than for charm than for beauty, is therefore expeted to result in a hierarchy in the $R_{\mathrm{AA}}$, smaller for light hadrons than for charm than for beauty. ALICE has measured the $R_{\mathrm{AA}}$ for (various) D mesons, which is similar at high $p_{\mathrm{T}}$ to the one of light quarks [5]. While this can be explained by the softer fragmentation and $p_{\mathrm{T}}$ spectrum of gluons with 

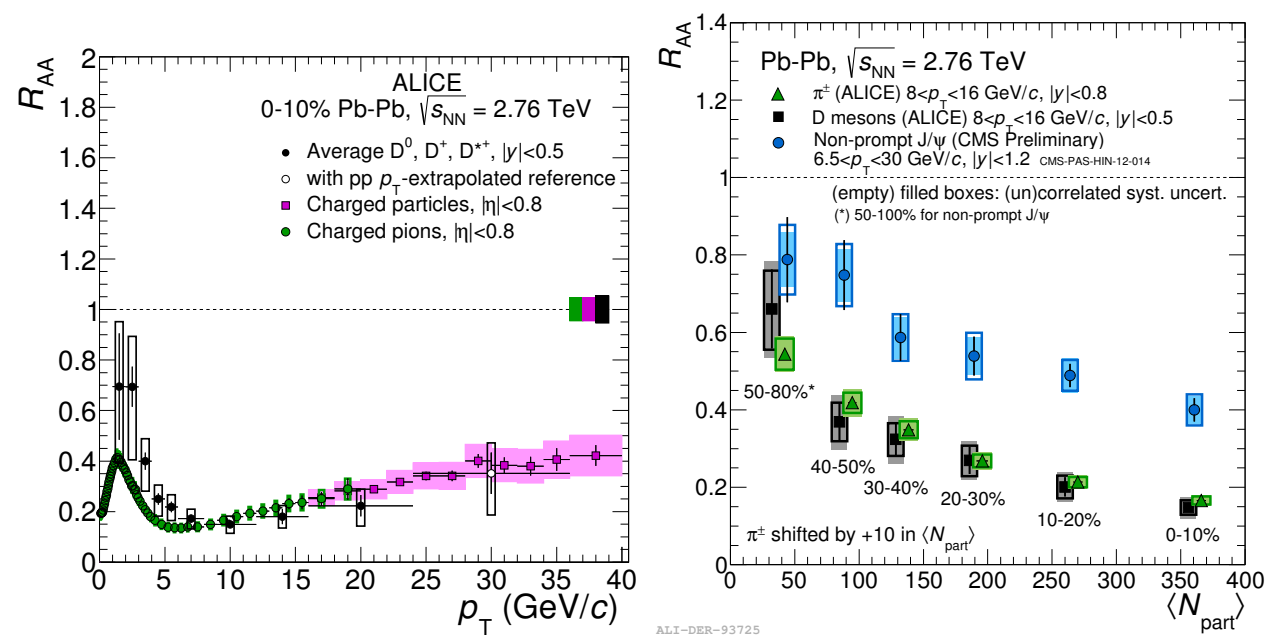

Figure 4. Left: Prompt D-meson $R_{\mathrm{AA}}\left(\right.$ average of $\mathrm{D}^{0}, \mathrm{D}^{+}$and $\mathrm{D}^{*+}$ ) as a function of $p_{\mathrm{T}}$ compared to the nuclear modification factors of pions and charged particles in the $0-10 \%$ centrality classes. Right: Comparison of the D meson $R_{\mathrm{AA}}$, to the charged pion $R_{\mathrm{AA}}$ integrated over $8<p_{\mathrm{T}} \tilde{<} 16 \mathrm{GeV} / \mathrm{c}$ and of the preliminary $R_{\mathrm{AA}}$ of non-prompt $\mathrm{J} / \psi$ mesons in $6.5<p_{\mathrm{T}} \tilde{<} 30 \mathrm{GeV} / \mathrm{c}$ measured by the CMS experiment.

respect to those of c quarks, there are indications at low $p_{\mathrm{T}}$ of $R_{\mathrm{AA}}(\mathrm{D})>R_{\mathrm{AA}}(\pi)$. The hierarchy between charm and beauty is tested instead with the comparison between $\mathrm{D}$ and secondary $\mathrm{J} / \psi$ (from $\mathrm{B}$ decays) measured by CMS for central collisions, where the expected hierarchy of $R_{\mathrm{AA}}(\mathrm{c})<R_{\mathrm{AA}}(\mathrm{b})$ is observed.

\section{The quarkonium saga}

One of the very early signature for the discovery of the QGP was suggested to be the suppression of quarkonia, tigthly bound states of charm or beauty quark and its anti-quark. Debye screening of the color fields in the medium would prevents binding in the QGP because the color charge of one quark is masked by the surrounding medium. A sequential melting of the quarkonium states would be observed as a result of their different binding energies On the other hand it became soon clear that a completely different scenario may occur, under the name of recombination, which becomes more and more relevant with increasing collision energy which in turn increases the cc pair multiplicity: the $\mathrm{J} / \psi$, melted in the QGP or eventually never really formed, may (re)generate from independently produced cc quarks at freeze out, therefore leading to an enhancement of $\mathrm{J} / \psi$ (or less suppression). While the data collected so far indicate that the suppression measured at $\sqrt{s_{\mathrm{NN}}}=2.76 \mathrm{GeV}$ is smaller, has a weaker centrality dependence, and a stronger momentum dependence than what measured at RHIC at $\sqrt{s_{\mathrm{NN}}}=200 \mathrm{GeV}$, which is very suggestive of the recombination scenario, the energy increase of Run 2 span will provide both a higher color screening, leading to more suppression, and a higher charm cross section, leading to more regeneration.

The statistics collected in $\mathrm{Pb}-\mathrm{Pb}$ collisions at $\sqrt{s_{\mathrm{NN}}}=5.02 \mathrm{TeV}$ is large and allows a more precise study than in Run1, in particular more narrow centrality bins and larger $p_{\mathrm{T}}$-reach [6]. The data collected in pp collisions at the same energy provides a reference, which is in very good agreement with the interpolation values used until now. Figure 5 shows a clear suppression with almost no centrality 

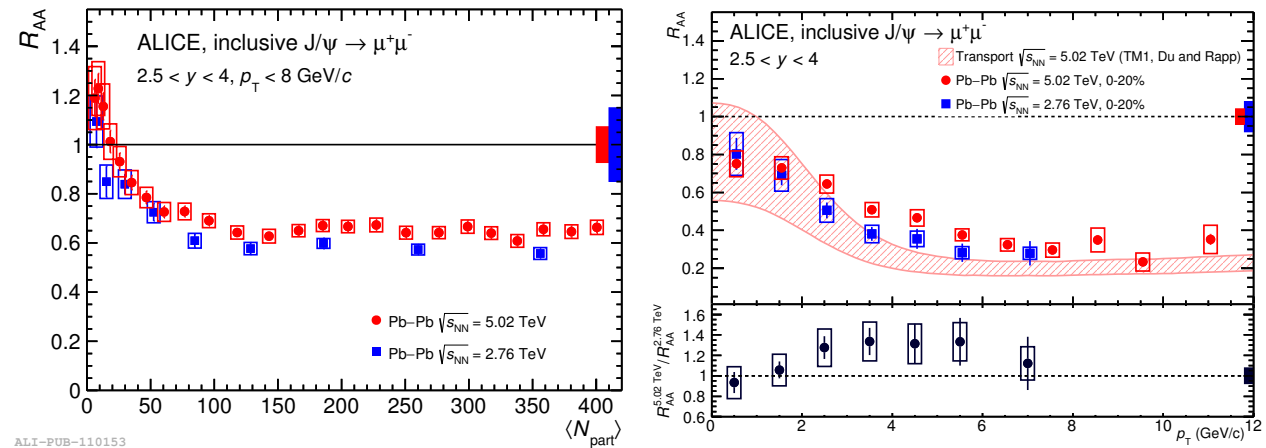

Figure 5. Left: The nuclear modification factor for inclusive $\mathrm{J} / \psi$ production, as a function of centrality, at $\sqrt{s_{\mathrm{NN}}}$ $=5.02 \mathrm{TeV}$, compared to published results at $\sqrt{s_{\mathrm{NN}}}=2.76 \mathrm{TeV}$. Right: The $p_{\mathrm{T}}$ dependence of the inclusive $\mathrm{J} / \psi$ $R_{\mathrm{AA}}$ at $\sqrt{s_{\mathrm{NN}}}=5.02 \mathrm{TeV}$, compared to the corresponding result at $\sqrt{s_{\mathrm{NN}}}=2.76 \mathrm{TeV}$ and to the prediction of a transport model (TM1), in the centrality interval $0-20 \%$. The $p_{\mathrm{T}}$ dependence of the ratio is also shown.

dependence above $N_{\text {part }} 100$. There is a systematic difference of approximately $20 \%$ with respect to the data at $\sqrt{s_{\mathrm{NN}}}=2.76 \mathrm{TeV}$, which however is within the total uncertainty of the measurements. The data are compared to various models, among which the statistical model, where $\mathrm{J} / \psi$ are created at chemical freeze-out, as well as to transport models, one (TM1), where $\mathrm{J} / \psi$ are created following a thermal rate equation and which imprements dissociation and regeneration in QGP and in the hadronic phase; another one (TM2) which describe the medium evolution with hydrodynamics, and finally to the 'co-mover' model where $\mathrm{J} / \psi$ are dissociated via interactions with partons/hadrons in the same $\mathrm{y}$ range. While in general the models suffer of large uncertainties due to the choice of input parameters in particular cc cross section, for most calculations a better agreement is found when considering their upper limit which, for transport models corresponds to the absence of nuclear shadowing, which can be clearly considered as an extreme assumption. The double ratio allows for (some) error cancellation, especially in the models. However data are, within uncertainties, compatible with the theoretical models. We also study the $p_{\mathrm{T}}$ dependence, now extended up to $12 \mathrm{GeV} / \mathrm{c}$, also shown in Fig. 5. The $R_{\mathrm{AA}}$ shows less suppression at low with respect to high $p_{\mathrm{T}}$, with stronger $p_{\mathrm{T}}$ dependence for central events as expected from models with strong regeneration component. There is a hint for an increase of $R_{\mathrm{AA}}$ with $\sqrt{s_{\mathrm{NN}}}$ visible in $2<p_{\mathrm{T}}<6 \mathrm{GeV} / \mathrm{c}$, while they are consistent elsewhere. In general models do not have the sensitivity to discriminate the different scenarios for the energy increase.

\section{The control experiment: benchmarks and surprises}

The studies of hard probes are typically based on the comparison between the data from $\mathrm{pp}$ and $\mathrm{Pb}-\mathrm{Pb}$ collisions, and attribute the suppression of the yields observed in $\mathrm{Pb}-\mathrm{Pb}$ to energy loss in the medium created in the final state. A control experiment is necessary to disentangle strong final state effects from initial state effects, due to the presence of the nuclei themselves and not the QGP. This control experiment may come from either from the measurement of electroweak probes, that do not experience strong interaction withe the medium, or from the measurements in $\mathrm{p}-\mathrm{Pb}$ collisions where only cod nuclear matter is created. 

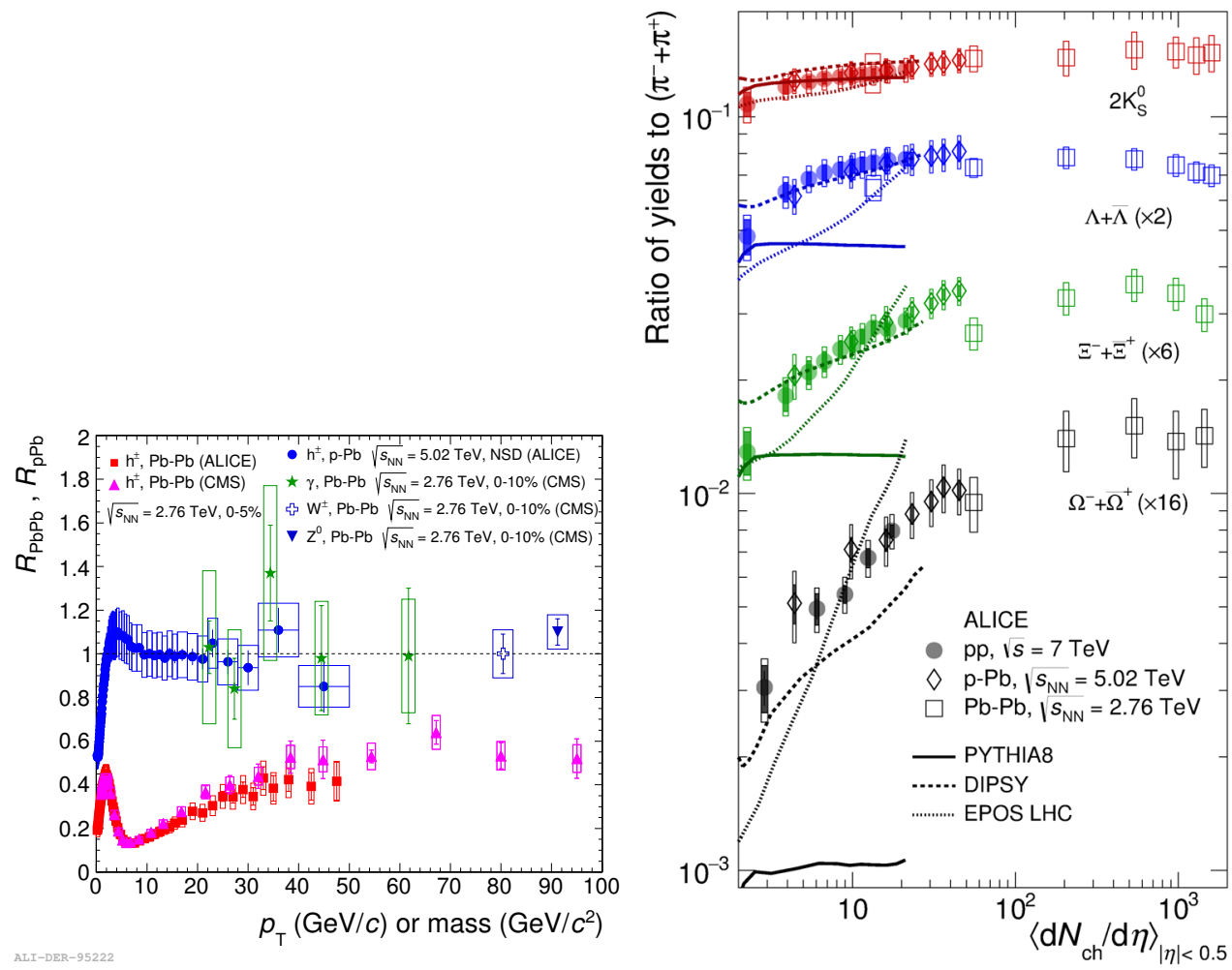

Figure 6. Left: $R_{\mathrm{AA}}$ of charged particles of a function of $p_{\mathrm{T}}$ measured in minimum-bias $\mathrm{p}-\mathrm{Pb}$ collisions at $\sqrt{s_{\mathrm{NN}}}=5.02 \mathrm{TeV}$ in comparison to data in central $\mathrm{Pb}-\mathrm{Pb}$ collisions at $\sqrt{s_{\mathrm{NN}}}=2.76 \mathrm{TeV}$ for charged particle, direct photon, $\mathrm{Z}^{0}$ and $\mathrm{W}^{ \pm}$production. Right: $p_{\mathrm{T}}$-integrated yield ratios of strange and multi-strange hadrons to pions as a function of $\left\langle\mathrm{d} N_{\mathrm{ch}} / \mathrm{d} \eta\right\rangle$ measured in the rapidity interval $|y|<0.5$ in $\mathrm{pp}, \mathrm{p}-\mathrm{Pband} \mathrm{Pb}-\mathrm{Pbcollisions}$, compared to calculations from MC models of pp collisions.

\subsection{Benchmarking hard probes}

ALICE measured the $p_{\mathrm{T}}$ of charged hadrons in $\mathrm{p}-\mathrm{Pb}$ collisions and the nuclear modification factor $R_{\mathrm{pA}}$ consistent with unity [7]. No suppression is observed also for the $R_{\mathrm{pA}}$ of heavy flavor [8] and jets [9]. This may indicate the absence of cold nuclear matter effects, however calculations with models that include moderate initial or final state effects can reproduce the data. These benchmark measurements in $\mathrm{p}-\mathrm{Pb}$ collisions, together with the measurement of electroweak probes, measured by $\mathrm{CMS}$, such as the direct photons [10], and the weak bosons $\mathrm{W}^{ \pm}[11]$ and $\mathrm{Z}^{0}$ [12] (see Fig.6) confirm the validity of the $N_{\text {coll }}$ binary scaling, and therefore provide the experimental demonstration that the suppression observed in $\mathrm{Pb}-\mathrm{Pb}$ collisions is due to parton energy loss in a hot QGP.

\subsection{Collectivity in small systems}

However, while the measurement of benchmark processes has served as an important baseline for the understanding and the interpretation of the $\mathrm{Pb}-\mathrm{Pb}$ data, several measurements, especially in the low and intermediate $p_{\mathrm{T}}$ region, could not be explained by an incoherent superposition of pp collisions, but 
instead are compatible with the presence of coherent and collective effects, whose strength increases with multiplicity. ALICE has performed various studies of multiplicity dependence of particle production, in $\mathrm{pp}, \mathrm{p}-\mathrm{Pb}$, and $\mathrm{Pb}-\mathrm{Pb}$ collisions. Of particular interest are the measurements of strange and multi-strange hadrons, whose hierarchical enhanced production in AA collisions was one of the earliest proposed indicators for the formation of the QGP state and, although observed in collisions of heavy nuclei at SPS, RHIC and LHC, is not yet understood at fundamental level. The right panel of Fig. 6 shows the ratios of strange hadron to pion yields as a function of $\left\langle\mathrm{d} N_{\mathrm{ch}} / \mathrm{d} \eta\right\rangle$ in $\mathrm{pp}, \mathrm{p}-\mathrm{Pb}$, and $\mathrm{Pb}-\mathrm{Pb}$ collisions [13]. A significant enhancement of strange to non-strange hadron production is observed with increasing particle multiplicity, already in pp collisions. The relative increase is more pronounced for $\Omega$ than for $\Xi$, indicating that the strangeness content may control the rate of increase with multiplicity. The behaviour $\mathrm{pp}$ collisions resembles that of $\mathrm{p}-\mathrm{Pb}$ collisions, both in the values of the ratios and in their evolution with the event activity, and, at the highest multiplicities, reach the values measure in $\mathrm{Pb}-\mathrm{Pb}$ collisions, which were shown to be compatible with the Gran Canonical limits. This suggests that the origin of strangeness production in hadronic collisions is driven by the characteristics of the event activity rather than by the initial-state collision system or energy.

\section{Summary}

Heavy ion collisions produce the most extreme state of matter ever created in the laboratory The AA physics program is rich but still full of open questions. Quantitative understanding requires not only a firm baseline from $\mathrm{pp}$ measurements, but also constrains of cold nuclear matter effects from $\mathrm{p}-\mathrm{Pb}$ data. While baseline measurements provide clear proof that effects observed in $\mathrm{Pb}-\mathrm{Pb}$ collisions are genuine effects related to parton energy loss in the hot deconfined QCD matter, various measureuments have been performed, suggestive of the existence of collective effects at high multiplicities also in small systems. ALICE has collected an excellent set of data for $\mathrm{pp} \mathrm{p}-\mathrm{Pb}$ and $\mathrm{Pb}-\mathrm{Pb}$ collisions both in Run 1 and Run2 which allow a significant progree in the precision measurements of the QGP properties.

\section{References}

[1] B. Muller, J. Schukraft, and B. Wyslouch, Ann. Rev. Nucl. Part. Sci. 62 (2012) 361-386.

[2] ALICE Collaboration, Phys. Rev. Lett. 116 (2016) 222302.

[3] ALICE Collaboration, Phys. Rev. Lett. 116 (2016) 132302.

[4] ALICE Collaboration, Phys. Lett. B 753 (2016) 319-329.

[5] ALICE Collaboration, JHEP 03 (2016) 081.

[6] ALICE Collaboration, arXiv:1606.08197.

[7] ALICE Collaboration, EPJC 74 (2014) 3054.

[8] ALICE Collaboration, Phys. Rev. Lett. 113 (2014) 232301.

[9] ALICE Collaboration, Eur. Phys. J. C 76 (2016) 5, 271.

[10] CMS Collaboration, Phys. Lett. B 710 (2012) 256.

[11] CMS Collaboration, Phys. Lett. B 715 (2012) 66.

[12] CMS Collaboration, Phys. Rev. Lett. 106 (2011) 212301.

[13] ALICE Collaboration, arXiv: 1606.07424. 